\title{
HIGH CURRENT MAGNETIZED PLASMA DISCHARGE
AND ELECTRON BEAMS FOR CAPTURE AND COOLING OF CHARGED LEPTONS AND HADRONS
}

\author{
Ady Hershcovitch \\ Brookhaven National Laboratory, Upton, N.Y. 11973, USA
}

\section{Abstract}

Nowadays most magnetic lenses used to capture and to focus pions and muons utilize azimuthal magnetic fields generated by large axial currents, like horns or lithium rods (or even a Z-pinch at GSI). Capture and focusing angle is proportional to the product of the current and length of the lens. State-of-the-art for these lenses is no more than $750 \mathrm{kA}$ and $70 \mathrm{~cm}$. A meter long, multi-MA, magnetized axial discharges were generated in the early days of fusion. Lenses based of such devices can increase the capture angle of pions, e.g., by more than a factor of 2 . Electron beam cooling is presently achieved in storage rings by having charged particles interact with a comoving electron beam. In these devices, typical parameters are electron beam currents of about $1 \mathrm{~A}$, an interaction length of about 1 meter, and interaction time of about $30 \mathrm{msec}$. Multi-MA electron beams can be used for single-pass final stage cooling in a number of machines. Calculations for some applications, as well as other advantages indicate that these schemes deserve further more serious consideration.

\section{INTRODUCTION}

\subsection{Focusing}

In many areas of research involving charged particle beams, various methods of magnetic focusing have been employed to enhance the flux of charged particles from a divergent source such as a production target,[1] or to confine ions emerging from the crossover region of an ion diode to betatron oscillation for propagation to a small target a few meters away.[2] The method of choice for focusing of high energy charged particles, produced in nanosecond to microsecond bursts, that need to be transported for a distance of a meter or more has been the use of azimuthal magnetic fields that pull the particles radially inward as a consequence of the Lorentz force. These fields are usually generated by currents that are oriented along the desired flight path of the charged projectiles.

Lithium lenses[3,4] and focusing horns[1,5] have been used in high energy physics research, while various spark and $Z$ channels were developed for fusion experiments. $[2,6]$

Although some features vary from experiment to experiment, there are a number of common requirements for all these lenses: 1). Very large axial currents must be generated and sustained. 2). The lens medium should have as low a density as possible to minimize particle absorption and scattering. 3). Due to large currents and high magnetic fields, the lens must withstand high mechanical and thermal stresses. 4). The lens must survive prolonged exposure to radiation.

\subsection{Cooling}

A low thermal spread electron beam moving at the same velocity with a hotter charged particle beam will have a cooling effect on that beam. Electron beams, and other discharges can be designed to have cold electrons moving at the same velocity as leptons and hadrons to cool them.

\section{PLASMA DISCHARGES OPTIONS}

Interest in charged particle focusing and/or cooling is varied and many of the broad number of applications require customized configurations. Evaluation of applicability of schemes, based on plasma devices, is done for the AGS muon $g-2$ experiment, a proposed AGS neutrino experiment, and the muon collider. These schemes do not have the drawbacks and limitation of presently used devices.

Requirements on horns to withstand very large thermal and mechanical stresses, and yet be fabricated from light elements to minimize particle losses, have limited their current to about $300 \mathrm{kA}$. The drawbacks of a lithium lens are due to the fact that lithium has very low mechanical strength, and it is very reactive chemically. Consequently, it must, however, be kept under high pressure in a strong container made of a chemically compatible material

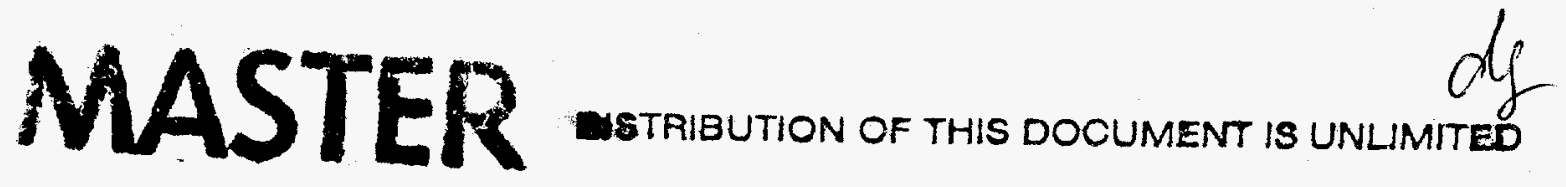


like titanium or stainless steel. Practical currents reached have been limited to about $500 \mathrm{kA}$.

Spark (or Z) channels are plasma transport channels, characterized by large currents (100's of $\mathrm{kA}$ ), which have been developed to transport intense beams of light ions over distances of up to 5 meters.[7] These channels consist of two annular plates placed in a vacuum chamber usually filled to a pressure of about 10 Torr. Pulse lengths of 10 's of nsec at a repetition rate of $500 \mathrm{~Hz}-1 \mathrm{kHz}$ have been generated, as well as three microseconds long pulses at lower repetition rates. Channel radii from $1 \mathrm{~cm}$ to more than $10 \mathrm{~cm}$ were reported.

Electron beam currents that are in the mega-Ampere range have been generated by diodes. Although most of these diodes operate with pulses that are in the nsec range, some diodes have operated with pulse lengths of up to 2 microseconds. Hollow-beam electron guns are particularly attractive due to their larger perveance and enhanced stability in addition to the obvious advantage of their hollow structure.

$\underline{Z}$ pinches involve a sudden compression of a low-density plasma by means of a large discharge current that lasts usually for up to a few microseconds. Its fill pressure is below a milli-Torr. Discharge currents of $10 \mathrm{MA}$ over a few centimeters have been reached. However, in the early days of fusion research with $Z$ pinches, experiments were performed with magnetized $Z$ pinches. In one device with an axial magnetic field of 1.5 tesla, $2 \mathrm{MA}$ were reached in a length of 0.8 meters for 250 microseconds.[8] This approach failed for fusion, since in that device little pinching occurred (the ion temperature was only $50 \mathrm{eV}$ ). But, these early devices reached parameters which make them ideal candidates for lepton and hadron lenses.

\section{NOVEL PION AND MUON LENSES}

Requirements for first focusing lens of the neutrino oscillation experiment are a 0.2 meter long lens with a current of $2.8 \mathrm{MA}$ pulsed at the AGS cycle for 2.5 microseconds, and a $10 \mathrm{~cm}$ diameter.[9] Similar requirements exist for the g- 2 experiment with regard to the current and length combination of the lens, however, there is a preference for shorter pulse lengths $(10$ 's of nsec) at a higher repetition rate. A pion acceptance angle can be calculated from Danby's formula,

$$
\mathrm{p} \cdot \sin (\partial)=(\mathrm{B} \cdot \mathrm{L}) / 1313.22
$$

Where $p$ is particle momentum in $\mathrm{GeV} / \mathrm{c}, \mathrm{B}$ is magnetic field in $k G$, and length $L$ in inches.

The magnetic field is determined from Ampere's Law which for uniform current distribution at a radial distance $r$ yields

$$
\mathrm{B}=0.2 \cdot \mathrm{I} / \mathrm{r}
$$

Where the units for B, I, and $\mathrm{r}$ are Gauss, Amperes, and centimeters, respectively.

A wall-stabilized $Z$ pinch with a magnetic field reached the following parameters 40 years ago [8]:

0.8 meter long discharge with a current of $2 \mathrm{MA}$ for a duration of $250 \mu \mathrm{sec}$ was achieved. The axial magnetic field was 1.5 Tesla and the discharge radius was about $6 \mathrm{~cm}$. A lens based on such a discharge yields, from equations (1) and (2), an acceptance angle of at least 0.263 radians (the axial magnetic field enhances the acceptance). Tapering the discharge can further enhance pion focusing for these experiments.

For the muon collider, a magnetized $Z$ pinch with a current of $10 \mathrm{MA}$ was considered. A preliminary computation[10] for the focusing capability of a lens based on a series of $10 \mathrm{MA} \mathrm{Z}$ pinches, which were equivalent in size and length to conventional lenses, was performed with a computer code that was developed by J. Gallardo and R. Palmer. The production target was bombarded by 10 $\mathrm{GeV}$ protons yielding pions with energies of $1.2 \mathrm{GeV}$ $\pm 0.98 \mathrm{GeV}$, which later decay into $0.8 \mathrm{GeV} \pm 0.73$ $\mathrm{GeV}$ muons. The muon-to-proton ratio for this system of lenses is $\mu / p=0.38$. This represents a $50 \%$ enhancement in muon capture when compared to two other scenarios that utilize similar configurations. For example, lithium lenses yielded $\mu / p=0.23$

\section{SINGLE PASS ELECTRON COOLING}

At first glance, this idea does not seem feasible with untrapped particles. However, if the parameters from IEAR are scaled up, this idea seems more interesting. At LEAR,[11] a 1 Ampere electron beam will cool antiprotons in 0.03 seconds if exposed continuously to the electron beam. Thermal equilibration time is

$$
\tau=5.56 \times 10^{18} \frac{\left(m_{h} T_{c}+m_{c} T_{h}\right)^{3 / 2}}{\left(m_{c} m_{h}\right)^{1 / 2} z_{h}^{2} z_{c}^{2} \lambda n_{c}} \mathrm{sec}
$$

given by (eV, cgs units) [12]

Where subscripts $c$ and $h$ refer to cold and hot particles, respectively. It is clear from Equation 3 
that equilibration time is proportional to the density of the lower temperature particles and for electron beams with equal cross section (and velocity), the electron density is proportional to the current. To scale up from LEAR, equation 3 indicates that a 3 meter long $1 \mathrm{MA}$ electron beam can cool a muon in its transit time of about 10 nsec.

If pions or muons are initially cooled by other means, electron cooling can provide final stage cooling for the muon collider, e.g., a muon beam, that was cooled and slowed down to a momentum $P$ $=300 \mathrm{MeV} / \mathrm{c}$ and a momentum spread of $\Delta \mathrm{p} / \mathrm{p}=4 \%$, can be further cooled by a $10 \mathrm{MA}, 1.68 \mathrm{~m}$. long, 1 $\mathrm{cm}$. radius electron beam (with $0.516 \mathrm{MeV}$ electrons) to $\Delta \mathrm{p} / \mathrm{p}=2.86 \times 10^{-3}$. A second stage of $10 \mathrm{MA}, 56$ $\mathrm{cm}$. long electron beam reduces $\Delta \mathrm{p} / \mathrm{p}$ to $1.53 \times 10^{-5}$ .[13]

In cooling $\mu-$, the magnetic field generated by the co-moving electrons focuses $\mu-$. But, such a magnetic field defocuses $\mu^{+}$. To cool $\mu^{+}$particles, no net axial current should exist in a channel. One possibility is to shoot the $10 \mathrm{MA}$ electron beam through a $10 \mathrm{MA} Z$ pinch such that the two currents cancel each other.[13]

\section{CONCLUSIONS}

Evaluation of pion and muon capturing and focusing capability of a lens, based on parameters which were achieved in a magnetized $Z$ pinch 40 years ago ( $\mathrm{MA}, 0.8 \mathrm{~m}$. long, $6 \mathrm{~cm}$. radius), indicates that this lens performance can far exceed that of presently used lenses. Additionally, particles focused in a spark channel or a $Z$ pinch lens travel in what is essentially vacuum, hence there is indeed negligible absorption and scattering of pions or muons. By comparison, state-of-the-art performance for a horn is $300 \mathrm{kA}$, while peak performance of a typical lithium lens is at about $500 \mathrm{kA}$. Since a lithium lens has a radius of 1 $\mathrm{cm}$ or smaller, the magnetic field at a distance of about $10 \mathrm{~cm}$ from the lens axis (where it is most important for focusing) is too low to be of interest for the AGS neutrino oscillation experiment and the g-2 experiments, for example. Thirty years ago, a very modest version of such a lens was used in an AGS experiment. This lens performed well until a ceramic liner broke and was not replaced since the experiment was close to its conclusion.[14] Similarly, a series of lenses based on magnetized $Z$ pinches could greatly improve pion capture and focusing, for the muon collider, over presently pursued schemes.

Single pass final stage cooling with very intense electron beams, suitable for the muon collider or between accelerators and storage rings, does not seem unreasonable. However, there is no data base which suggests that generating the needed electron beams is straight forward.

Multi-Mega-Ampere $Z$ pinches deserve a further, more serious consideration for possible use as lenses in various experiments, and in the muon collider. State-of-the-art $\mathrm{Z}$ pinches have reached 10 $M A$ with vastly improved capacitor technology. Extending a $Z$ pinch type discharge length and radius with wall and axial magnetic field stabilization is possible. This will result in a larger current as well. Consequently, pursuing this approach can result in a formidable pion capturing and focusing lens.

\section{ACKNOWLEDGMENTS}

Work performed under the auspices of the U.S. Department of Energy. The author is grateful to J. Gallardo, R. Palmer, and A. Bogacz for many useful discussions. Computer calculation performed by J. Gallardo is gratefully acknowledged.

\section{REFERENCES}

[1] M. Giesch, et al., NIM 20, 58 (1963).

[2] C.L. Olson, J. Fusion Energy 1, 309 (1982).

[3] G. Dugan, et al., IEEE Trans. Nucl. Sci. NS-30, 4, 3660 (1983).

[4] A. Ijspeert and P. Sievers, CERN SPS/86-18 (ABT).

[5] A. Carroll, et al., Proceedings of IEEE Particle Accelerator Conf. 1987, p. 1731 .

[6] J. Olsen and R. Leeper, J. Appl. Phys. 53, 3397 (1982).

[7] P. Ottinger, et al., Proceedings of IEEE, 80,1010 (1992).

[8] A series of papers (by Golovin, et al., Komelkov, et al., and Andrianov, et al.), presented at the second U.N. Conf. on Peaceful Uses of Atomic Energy, Vol. 31 and 32, Geneva, 1958.

[9] A. Carroll, private communication, 1994; G. Bunce, private communication, 1994.

[10] A. Hershcovitch, Some Options for Pion and Muon Focusing in the AGS $\mathrm{g}-2$ and Neutrino Oscillation Experiments (and Possible New Focusing and Cooling Schemes for a Muon Collider), AGS/AD Tech.Note 413 (1995).

[11] H. Poth, CERN-EP/90-04; H. Poth, et al., NIM A287, 328 (1990).

[12] B.A. Trubnikov, Particle Interactions in a Fully Ionized Plasma, Rev. of Plasma Phys., I (Consultants Bureau, N.Y.) 105 (1965).

[13] Muon Muon Collider: Feasibility Study, BNL - 52503, FNL - Conf. 96/092. LBNL - 38946, July 1996, Ch. 12.

[14] E.B. Forsyth, L. Lederman, and J. Sunderland, IEEE Trans. Nucl. Sci., p. 872 (June, 1965); E.B. Forsyth, private communication, 1995. 


\section{DISCLAIMER}

This report was prepared as an account of work sponsored by an agency of the United States Government. Neither the United States Government nor any agency thereof, nor any of their emplcyees, makes any warranty, express or implied, or assumes any legal liability or responsibility for the accuracy, completeness, or usefulness of any information, apparatus, product, or process disclosed, or represents that its use would not infringe privately owned rights. Reference herein to any specific commercial product, process, or service by trade name, trademark, manufacturer, or otherwise does not necessarily constitute or imply its endorsement, recommendation, or favoring by the United States Government or any agency thereof. The views and opinions of authors expressed herein do not necessarily state or reflect those of the United States Government or any agency thereof. 


\section{DISCLAIMIER}

Portions of this document may be illegible in electronic image products. Images are produced from the best avaliable original document. 